\title{
ERP processes automation in corporate environments
}

\author{
Victor Antonoaie ${ }^{1}$, Adrian Irimeş $^{2}$, and Lucia-Antoneta Chicoş ${ }^{3, *}$ \\ ${ }^{1}$ Transilvania University of Braşov, Department of Management and Economic Informatics, Colina \\ Universităţii 1, Brașov, Romania \\ ${ }^{2}$ IBM Romania, Turnului 5, Braşov, Romania \\ ${ }^{3}$ Transilvania University of Braşov, Department of Manufacturing Engineering, Mihai Viteazul 5, \\ Braşov, Romania
}

\begin{abstract}
The automation processes are used in organizations to speed up analyses processes and reduce manual labour. Robotic Automation of IT processes implemented in a modern corporate workspace provides an excellent tool for assisting professionals in making decisions, saving resources and serving as a know-how repository. This study presents the newest trends in process automation, its benefits such as security, ease of use, reduction of overall process duration, and provide examples of SAPERP projects where this technology was implemented and meaningful impact was obtained.
\end{abstract}

\section{Automation principles}

The IT industry has, for decades, focused on process automation and ways to increase IT productivity and efficiency with aim to reduce operator errors. Due to market condition, the need to provide information and faster speed to market, the focus is shifting to enhance business productivity. The goal for these improvements is to help capture new markets and gain a competitive edge in today's business and technology race. In order to achieve these goals, the company needs to drive down costs and improve efficiency, to free funds and resource to make strategic investments. Furthermore, making sure that the IT specialists are focusing on innovation in the base areas of the business, working through complex problems, and improving productivity is of the highest importance [1]. Thus, automation is becoming ever more critical.

Until now, traditionally, the automation process relies on scripts that combines computer commands that are designed for a single platform or for a specific product. In order to achieve this, traditional process automation involves design and implementation of multiple instances of the same product. This type of automation is now becoming obsolete and modern methods are required. Now, solutions that use modern analytics-based automation are becoming the building block of the next generation enterprise IT system. One example is the IBM Enterprise IT Automation Services, which is seen deployed in various organizations, helping them to become industry leaders.

\footnotetext{
* Corresponding author: 1.chicos@unitbv.ro
} 
The standard IT setup of today's enterprise includes technologies from an array of vendors as well as various sourcing strategies and delivery models that span several platforms and products. This modern and complex IT environment, makes fixing operations issues, even small ones, a challenge. Even trivial tasks and problems caused by the simplest issues, such as user password management, grow out of control due to an error file that is too large to use, and due to this the time to identify and fix an error takes many hours. These types of events can cause interruption or in time severely degrade overall performance, and can as a result have an impact on the enterprise business revenue.

The topic of this paper is to present the newest trends in process automation, its benefits (security, ease of use, reduction of overall process duration) and provide examples of ERPSAP projects where this technology was implemented and meaningful impact was obtained.

\section{Implications and uses of automation technology in the IT sector}

\subsection{What is Enterprise IT Automation services?}

Regardless of how good and solid the enterprise IT infrastructure is, how thorough and proactive the maintenance plans it has employed, problems with inevitably occur. Companies are dependent on IT staff to manage the errors, which itself is a risk. Well established processes and trimmed staffing models no not fully eliminate the time wasted as IT technicians respond to incidents and requests. Considering the criticality of certain incidents types the failure to solve them in time can have a significant business impact. To minimize the delay between the time of the failure and the action taken to fix the problem is to have in place autonomous software solutions, robots that monitor the enterprise IT landscape and that takes actions when something breaks.

Enterprise IT Automation Services provides the ability to fix problems immediately, as they occur, reducing the Mean Time to Respond (MTTR) from days and hours to minutes. By doing so, it prevents severity 3 and 4 events from becoming severity 1 events.

The enterprise IT infrastructure is continuously being analyzed and logged by Enterprise IT Automations Services, tracking and logging everything and so providing granular, transparent and deep level details. The created data can be then analyzed further in order to identify patterns and provide starting points for continuous improvements.

\subsection{What are the benefits of Enterprise IT Automation services?}

Automation service solutions [2] have been deployed consistently trough the clients' landscape worlds wide and demonstrated value to clients in areas like:

- Service level and quality improvements - Virtual engineers are autonomous dispatched to address and fix issue as they occur. Enterprise IT automation services dramatically reduce the MTTR by removing the dependency on human engineers to handle a large volume of the issues signalized. The risk for human error is also mitigated for the problems where virtual engineers are programmed to fix.

- IT staff productivity and efficiency improvements - Provides enterprise with the ability to do more work with less resources. The Return of Investments (ROI) is calculated considering the existing baseline and the potential gain by automation. Parallel to this cost benefits arisen with improved service levels and greater availability. Another indirect benefit results from freeing IT operations resources. Technicians are no longer distracted by low service interruptions and they can concentrate on fixing complex problems more effectively. 
- Continual services improvements - Efficiencies and improvements vary from one implementation to another, based on the baselines and penetration rates. The persistent pattern, however, across all is an improvement in the form of reduced response and resolution time, improved quality of service, and a reduction in outages.

\subsection{How does Enterprise IT Automation services work?}

Enterprise IT Automation Services includes several key components [1]:

- The virtual engineer is a software component that uses algorithms to analyze and automatically take action on issues that can impact the business process, without any form of human intervention. The virtual engineer continuously monitors the system for problems for which it is programmed for, and when it spots such an issue, it goes through a series of well-defined programmed actions (in a logical preset order) that are specific to the situation in order to remediate the issue.

- Patterns describe the behaviour that virtual engineers must follow to respond to an incident. There are standard patterns, client-specific patterns, and new patterns that are developed based on insights discovered by analytics.

- Analytics helps to identify what can be automated in further deployments, monitor the system behaviour of the automation, learning from company global experience, and so on. Analytics is used to measure the effectiveness of automation and patterns and to provide information that can be used for new development, changes to the existing systems, or determining the root cause of problems.

- Enterprise IT Automation Services is deployed as a service on multiple platforms like the IBM SoftLayer ${ }^{\circledR}$ cloud infrastructure. Therefore, it requires minimal effort and infrastructure commitments on an organization's IT environment.

Fig. 1 provides a high level view of how IBM Enterprise IT Automation Services integrates with the IT environment of an organization enabling the virtual engineer to detect, diagnose and either fix the problem or escalate it to a human engineer for resolution.

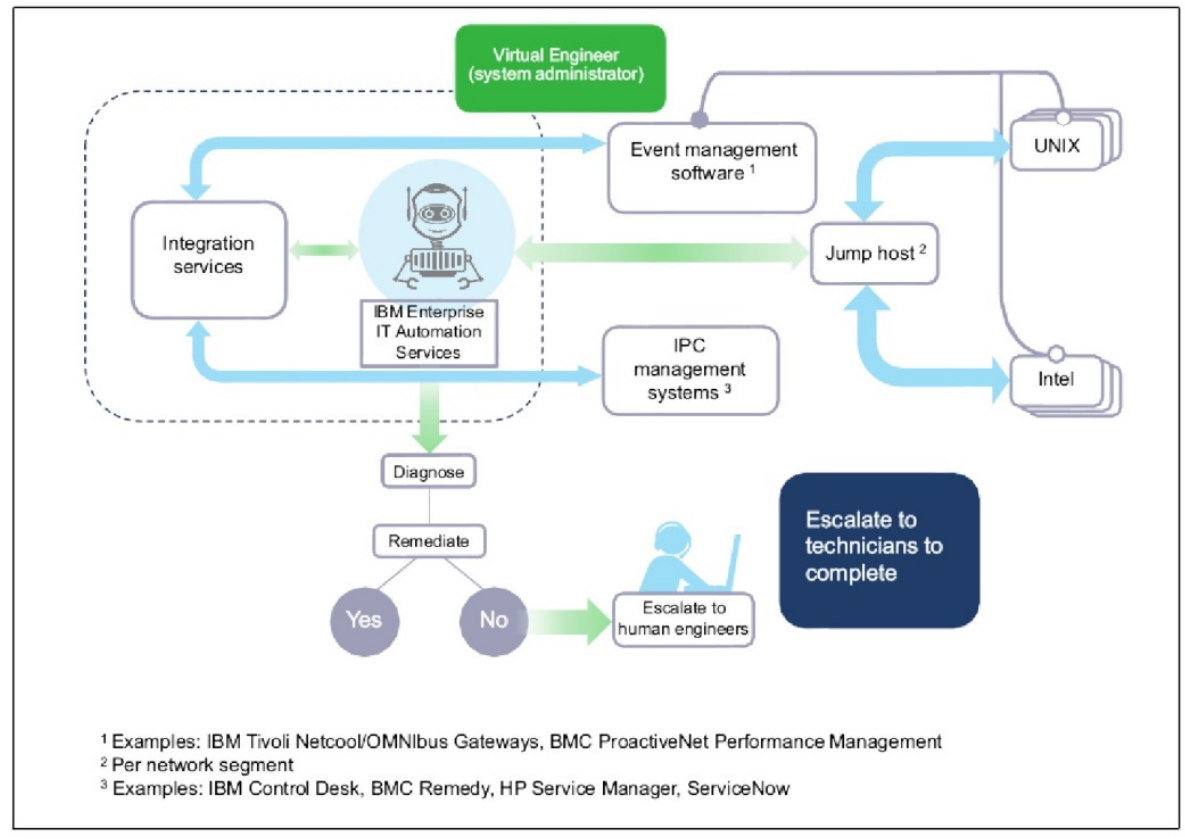

Fig. 1. Integration of IT Automation Services with the company IT environment [1]. 


\subsection{How are Enterprise IT Automation Services capabilities deployed in an enterprise IT environment?}

Enterprise IT Automation Services is deployed in two phases:

- Enablement

At first a onetime setup implementation is needed for the automation framework. Enterprise IT Automation Services slots into the enterprise IT operations as a new step in the support flows of IT technician's engagements. Enterprise IT Automation Services integrates into an organization's environment without requiring any changes to tools or previous automation investments. This is a simply and interface with key IT service management systems to automate use cases across the organization's IT infrastructure platforms, database, middleware and groupware applications, storage devices, network devices, and some business applications.

- Continual service improvement

A continual service improvement process based on analytics is instated to propose new automation use cases and updates to those deployed already by using:

- Trends in the behaviour of active (deployed) automation;

- New trends in the organization's environment, for example, new type of incidents, service requests and so on;

- Lessons learned from global implementations.

Are there any limits to what can be automated? Based on a clear set of questions, can be identified a system suitable for automation:

- Is the system on which automation should run reachable across the network?

- Is it possible to interact with the system programmatically by using APIs or shells?

- Is it possible to describe what have to be done on the system as a Standard Operating Procedure (SOP)?

If the answer is "yes" to all three questions, automation is applicable.

\section{The support system for an ERP implementation. Case Study - SAP implementation for the financial branch of an automotive company}

In this paper, the case study is structured into six main parts to better present the scale and complexity of an automation process implementation [3] into a live SAP ERP support system:

- Part 1. Scale of the implementation, integration with other applications, incident management systems, and general system description.

- Part 2. Distinguishing between the repetitive tasks, which can be automated, and difficult incidents which require a differential analysis and resolution. Automation advantages [4].

- Part 3. Designing an automation process guideline to perform the abovementioned tasks workflow. Complete tasks versus partial automation input.

- Part 4. Testing and implementation phase. Troubleshooting and process adjustment. Implementation in a live system.

- Part 5. Post implementation analysis - time and effort saved through automation. 


\subsection{Part 1 - System implementation and integration}

To be able to fully understand the need for an automation process [5] in an extended support system for an ERP (Enterprise Resource Planning) implementation we must first comprehend the scale and complexity of such an enterprise [6].

The case study selected by the authors focuses on a support system for a large ERP Implementation for two Banking Systems part of an automotive consortium. The SAP (Systems, Applications and Products in Data Processing) system installed for the client is part of a larger application network which handles all the functions and aspects of the company. As far as the user base is concerned, the SAP implementation is used by over 2000 individual user and several 'technical users' (a technical user is a user account which is used for communication purposes between application as well as for specialized automated tasks and jobs which require a certain set of authorizations to ease the role assignment process and ensure clear usage parameters). The system functions on a 24/7 schedule, with no downtime, with unrestricted user access. Automated processes, tasks, file transfers and other activities, part of the IT workflow [7], which do not require direct input are scheduled, in general, outside working hours to maximize resource use (as with any application system, the server resources are limited. Therefore, some activities need to be scheduled by taking into account the overall resource use to minimize the risk of failure due to insufficient memory or lengthy run times) and to ensure minimal potential interference from other user related activities.

The system issues can be sorted by the way in which they are reported:

- issues signalled by the user: a problem encountered by a user in any part of the system, ranging from a locked transaction, errors in performing a task to system dumps. These issues are signalled to the support team through an incident management system. The support team must resolve the issue in a time frame established by the severity of the problem (from $2 \mathrm{~h}$ in the case of a critical incident to 3 days in case of a low impact issues).

- issues triggered by failed SAP monitor: the support team sets monitor applications for key jobs [8] and processes running in the system. When one of them fails, the monitor automatically creates an incident and the support team is notified

- issues detected by the support team as part of regular checks: the team carries out checks on key parts of the system at scheduled intervals to monitor complex activities which cannot be covered by a monitor (because they require in depth analysis).

- incidents reported by other support teams: a support team from another linked application signals an issue with a cross-application process which requires the intervention of the SAP support team to fix.

\subsection{Part 2 - Establishing tasks for processing by automated robot}

As we have described in the above subchapter, the incidents received by the SAP-ERP support team can be very different, ranging from basic restarts of tasks to complicated program code issues. Therefore, the first step in any automation implementation must be to distinguish which of these tasks can be subjected to processing by an automated robot and which can be handled directly by the human operators.

Most of the incidents reported directly by users generally concern difficult issues, from all aspects of the application and are in most cases different, both in cause and in resolution. In this case there is no suitable way in which to automate the resolution process. The effort involved in creating the framework for each individual situation would be highly time and resource consuming and the expected savings would not be enough sufficient to merit the investment [9]. 
On the other hand, incidents which are repeatable and which have a clear, well established resolution process are prime candidates for robotic automation. In this category we can include failed jobs which require a simple restart, incidents reported by application monitoring which occur from various reasons, issues with data files not received by an application caused by delays etc.

The clear definition of how an incident process suitable for automation, in the general consensus of the automation guidelines [10] is: an incident which has a clearly established cause, which contains clear and complete information, which is repeating frequently enough to warrant an automated solution and which can be resolved entirely (or the automation robot would ensure a sufficient contribution to the solution workflow) by an automated robot working on a designed framework and within the set parameters of the workflow.

Therefore, the main concern when deciding to implement an automated robotic solution in a support system is to have enough such incidents to sustain the investment. More clearly put, the time and effort saved by the robot must be greater than the cost of the investment.

The main advantages of implementing an automated robot for such actions as described above would be (Fig.2):

- An Automation Robot is consistent throughout the entire process, which means consistent $24 \times 7$ process execution depending on the design set by the users. Moreover, the set process steps are followed correctly every time, without fault and at the same time, the results are predictable with no errors;

- A process automated by a robot has the advantage of availability: it can function on a $24 \times 7$ schedule, without needing vacations, without low performance periods and can be constantly monitored through specialized tools and reports;

- Security is a key feature of automated processes because: the robot only performs what it is programmed to do as part of a workflow. It only has access to the systems for which is programmed to work on and has a full audit trail of all actions. Moreover, it does not mine or harvest data and the risk of problems/failures is reduced because it has key control points in the process definition;

- Efficiency is perhaps one of the most valuable features of automation: robots performs tasks quicker and with better cost efficiency than human resources while also handling peak periods without needing resource reassignment.

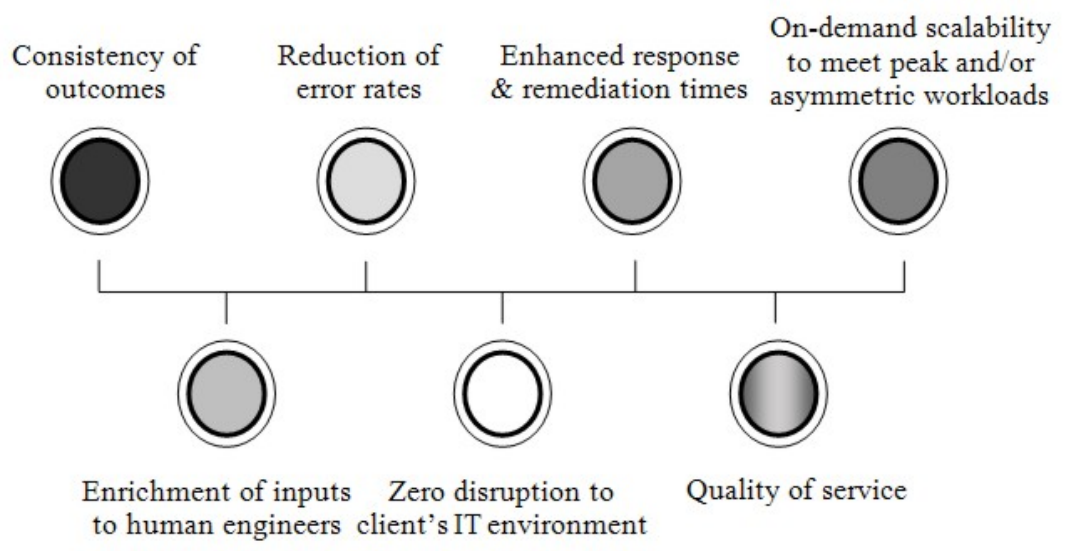

Fig. 2. Advantages of automation. 


\subsection{Part 3 - Designing automation process guideline}

Any automation process must start from an actual activity, performed by a human user within certain set parameters, such as: duration, complexity, number of repetitions within a time frame and costs.

After finding such an activity, like the ones presented in the subchapters above, the actual guideline process can begin. The first step is always to deconstruct the overall activity into steps arranged in the logical and chronological order. Then each individual step must be analyzed separately to determine the actions involved their duration and prerequisites as well as the effects.

Once each individual action is analyzed, measured and the robotic actions are determined, the individual action times are added up. The result is the overall robotic activity duration which is then compared to the time a human user would need to perform it. Nevertheless, this is not the only time consideration taken into account. For example, a human user would perform a system check three times during a normal work day of 8 hours, as these checks are only a small part of his or hers daily activities. On average, such a check would take approximately 10 minutes per activity which would generate a total of 0.5 hours per day. This is $6.25 \%$ of the workday dedicated to these checks. By comparison, an automated robot can do the same checks in less than 1 minute, ten times faster. Moreover, the robot can perform this task every hour, not only during working times but $24 \times 7$. This can prove very useful, as the more frequent the checks, the quicker a problem can be detected and resolved.

An automated robot [11] can perform any task he is designed to, as long as the initial programming includes all possible variables and it has clear and correct instructions. This is one of the most important parts of the activity workflow.

Nevertheless, there are instances where a process can be categorized and broken down into clear fixed steps only to a certain extent. As most SAP installations in companies contain large portions of customized code and programs, the number o variables involved in some processes can be too large to analyze and create scenarios on.

But this does not exclude automation completely from these workflows. If a problem resolution contains at least one initial standard step, that step can be automated. At first it might not seem as a necessary measure, even a time/effort saving of 5 minutes per incident can lead to a significant reduction of average solution times when looking at the entire company. The best example would be the automation for reading the information in a customer incident opened to the support team. The robot reads the text and searches for key words such as the target system, the client, transaction code used, error message code, etc., designed for each type of incident. If the robot does not find any of the key word in the message, it instantly e-mails the user and requests the missing information. If all the information required is present, the robot adds a line in the incident stating this fact which signals the human user that he can begin working on finding a solution to the issue.

\subsection{Part 4 - Testing and implementation of automation robot}

As with any new functionality to be implemented into a live production system, thorough testing and troubleshooting is required before the go live. Most SAP installations involve a three tier system, with a development system (where the programs are designed), an integration/testing system (which is an identical copy of the production system - it is here where programs are tested for functionality and potential issues) and a production system (a live system, where the users operate - and from where incidents will originate).

An automation robot implementation is no different from any other developed program or feature, therefore the first step in the installation is to apply the robots to the testing 
system. As this system is an identical copy of the production system, developers can generate the exact circumstances of a system issue and see how the robot performs.

It is also in this test phase where most of the adjustments can be made to the process and workflow, as even the best design needs real world issues testing to ensure complete functionality. This is also the stage where developers check if the robot is authorized to perform the designed tasks and can interact correctly with all the desired interfaces.

Successful test results in this pre-production system is mandatory before the automation robots can be implemented in the live system, as any potential flaw there could cause significant business impact.

When the automation robot receives the testing green light from all parties involved, specifically the developers, the business test team and the system administrator, in the next step is to install the automated solution in the production system.

The automation robot functions from a separate server and is not installed in the system. The robot functions in the system in much the same way in which a human user would. It would access it using preset credentials, follow SAP specific transaction patterns and perform general user actions.

\subsection{Part 5 - Post implementation analysis}

Even though the save in effort (FTE - Full Time Equivalent - the number of hours a human employee would work on a regular basis) and time is established in the early stages of the automation design, the real results can only be seen after the robot has been implemented in the production system and has been functioning for a longer period of time.

Based on the results of similar implementations in five different companies from various industries, the benefits of robotic automation implementation can be seen in table 1 and following charts (Fig.3 - 6).

Table 1. Analysis of automation by industry.

\begin{tabular}{|l|c|c|c|c|c|}
\hline $\begin{array}{c}\text { Company } \\
\text { Type/Indicator }\end{array}$ & $\begin{array}{c}\text { Telecommuni- } \\
\text { cation }\end{array}$ & $\begin{array}{c}\text { Energy } \\
\text { Distribution }\end{array}$ & $\begin{array}{c}\text { Financial } \\
\text { Services }\end{array}$ & $\begin{array}{c}\text { Technology } \\
\text { Company }\end{array}$ & $\begin{array}{c}\text { Large } \\
\text { Retailer }\end{array}$ \\
\hline $\begin{array}{l}\text { Total FTE } \\
\text { before } \\
\text { automation }\end{array}$ & 450 & 400 & 800 & 360 & 680 \\
\hline $\begin{array}{l}\text { Number of } \\
\text { processes } \\
\text { automated }\end{array}$ & 15 & 12 & 32 & 14 & 10 \\
\hline $\begin{array}{l}\text { Number of } \\
\text { robots }\end{array}$ & 160 & 120 & 180 & 130 & 168 \\
\hline FTE Saved & 300 & 250 & 360 & 210 & 342 \\
\hline $\begin{array}{l}\text { Projected ROI } \\
\%(\text { after 3 } \\
\text { years) }\end{array}$ & 80 & 65 & 85 & 72 & 59 \\
\hline
\end{tabular}




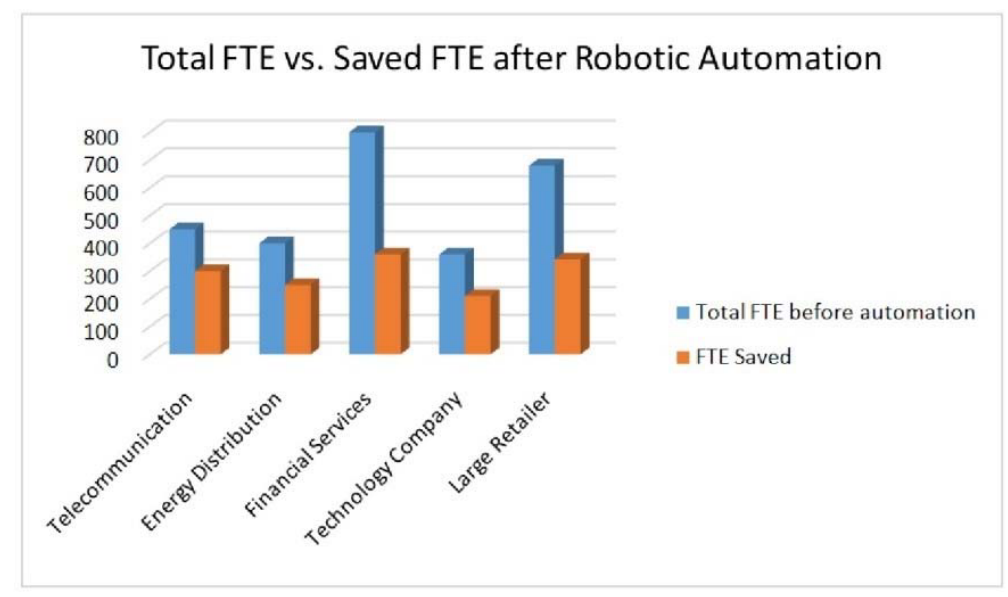

Fig. 3. Total FTE vs. saved FTE.

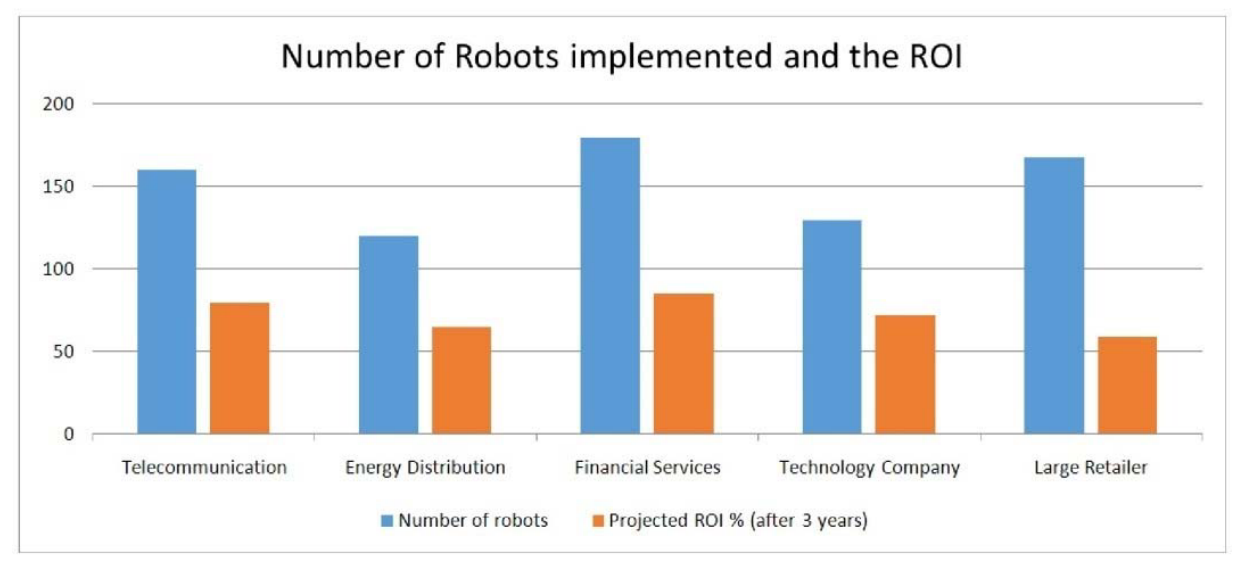

Fig. 4. Number of robots implemented and ROI.

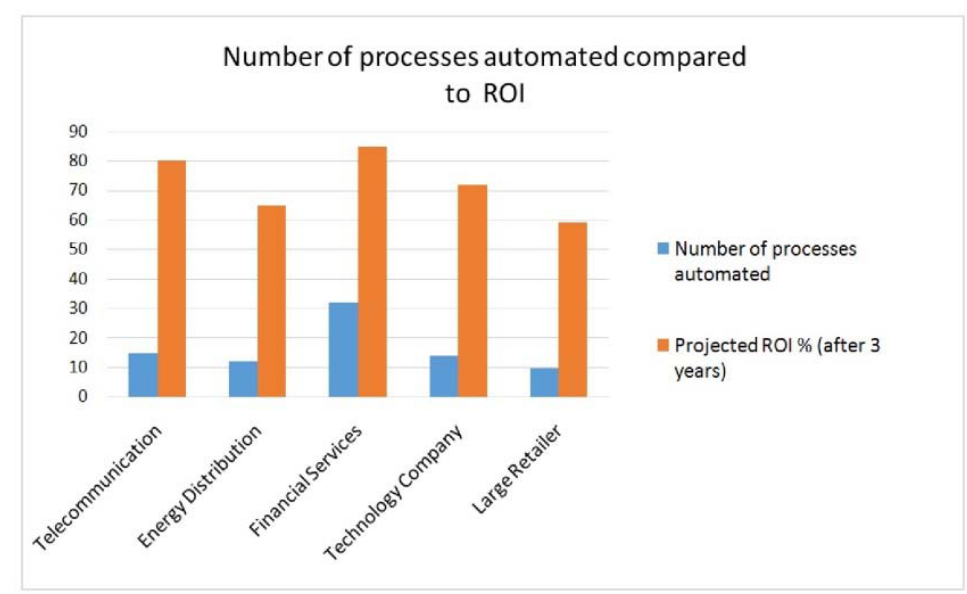

Fig. 5. Number of processes automated compared to ROI. 


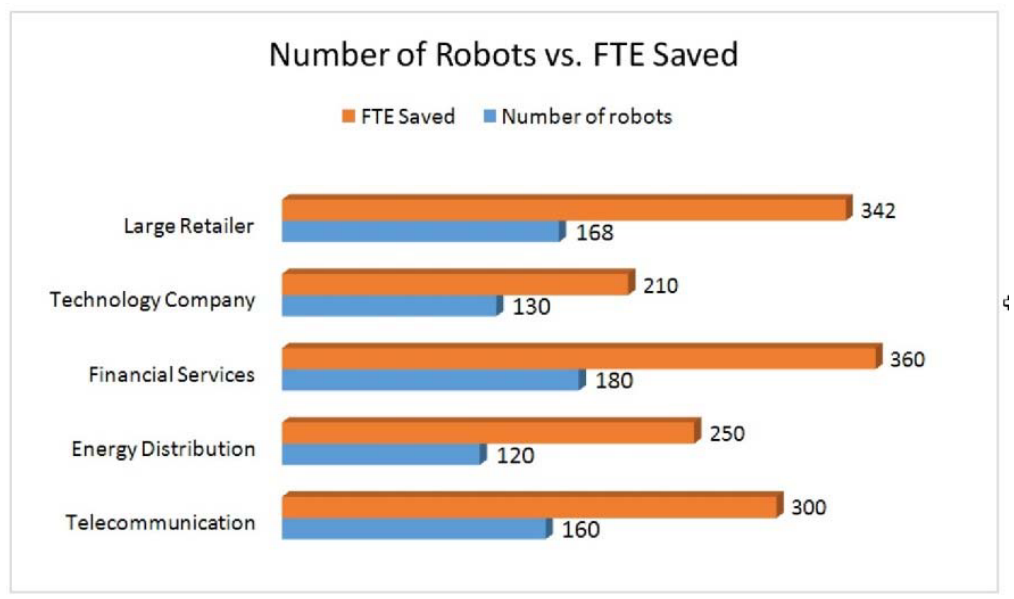

Fig. 6. Number of robots vs. FTE saved.

As it can be clearly deduced from the above charts, the advantages of processes automation are evident. There is a clear decrease in the FTE (Full Time Equivalent) after the automation robots took over certain task. Moreover, the more processes automated the greater the number of FTE saved. On top of that, the projected ROI (Return of Investment) increases proportionally to the number of company processes automated.

Automating processes with robots [12] is only one of the aspects of overall IT process optimization. To complement the work of automated robots in an IT company the future solution is the deployment of cognitive assistant machines [13] in these systems.

The cognitive assist is based on a super computer with a high capacity for assimilating and disseminating information. The processing power provided by this machine [14] is used to create a program which can answer real language questions based on previously registered information.

The application of such a machine in a SAP support system is mainly targeted on uploading operational manuals, problem resolutions, system designs and any other system related documentation. The purpose of this action is to create a large enough database to, potentially, allow for this cognitive assistant to provide solutions to issues based on similar problems faced in the past and system characteristics.

Even though such a task could take a long time to accomplish (taking into account the sheer volume of data needed to be inserted into the system) the results could prove extremely useful as the assistant could provide instant solutions to issue which otherwise would take hours/days to analyze. Moreover, the live database so created could provide a much more efficient way of storing and finding needed information compared to general database queries.

\section{Conclusions}

As it has presented in the above chapters of this papers, Robotic Automation of IT Processes is an inevitable step in the development of this ever-changing industry, as more and more companies have entered this field, both as providers of automated solutions as well as its recipients.

The advantages of such an implementation in a SAP-ERP system are clear and strong: reduction of response time, a cut in man hours spent on repetitive tasks, reduction of costs 
and avoidance of potential faults through human failure.

An automated robot is an excellent tool for assisting human operators in repetitive tasks as well as performing individual tasks. Moreover, when coupled with a well designed data base and a cognitive assistant, it can also serve as a live search data repository and perhaps event provide analytic solutions to system issues.

Even though the initial costs of automating suitable processes with robots are significant, both in terms of funding and effort in designing the workflow, the potential savings for the company are clear and failing to join the automation revolution could cost companies their business advantage in the future.

\section{References}

1. F. Bouz, H. Dave, IBM Enterprise IT Automation Services - Dynamic Automation in the Age of Cognitive, Dynamic Learning and Self-healing Systems (IBM Redbooks, NY, 2016)

2. M. A. Rochester, 5 Things to Know About IBM Enterprise IT Automation Services in the Age of Cognitive, Dynamic Learning and Self-healing Systems, https://www.ibm.com/developerworks/community/blogs/5things/entry/IBM_Dynamic_ Automation_in_the_Age_of_Cognitive_Dynamic_Learning_and_Self_healing_System s?lang=en, accessed 02.06 .2016

3. D. Krapohl, Integrated Approach to Organizational Development, http://www.augmentedintel.com/wordpress/index.php/integrated-approach-toorganizational-transformation/, accessed 10.06.2016

4. Process Automation. Streamline operations and increase productivity, http://www01.ibm.com/software/info/bpm/process-automation/, accessed 14.05.2016

5. B. Violino, Robotic process automation: The new IT job killer?,http://www.infoworld. com/article/2898108/robotics/robotic-process-automation-new-it-job-killer.html, accessed 2016

6. G. Nizri, IT Process Automation is the Way of the Future - Don't Get Left Behind! http://ayehu.com/it-process-automation-is-the-way-of-the-future-dont-get-left-behind/, accessed 10.06.2016

7. D. Georgakopoulos, M. Hornick, A. Sheth, An overview of workflow management: From process modeling to workflow automation infrastructure (Kluwer Academic Publishers, Boston, 1995)

8. A. Kento, Efect of Job Size Characteristics on Job Scheduling Performance. Job Scheduling Strategies for Parallel Processing, (Springer Berlin Heidelberg, 2000)

9. S.M.Merritt, J. L. Unnerstall, D. Lee, K. Huber, Hum Factors 57, 740 ( 2015)

10. B. Lydon, Industry 4.0 for Process Automation - Process Sensors 4.0 Roadmap, http:// www.automation.com/portals/process-automation/industry-40-for-process-automationprocess-sensors-40-roadmap, accessed 30.05.2016

11. What is Robotic Process Automation?, http://www.irpanetwork.com/what-is-roboticprocess-automation/, accessed 28.06.2016

12. D.Williams, IT Operations Run Book Automation, https://www.gartner.com /doc/492859/it-operations-run-book-automation, accessed 28.06.2016

13. D. A. Ferrucci, IBM J RES DEV 56, 1:1 (2012)

14. T. Winograd, R. Flores, Understanding Computers and Cognition (Ablex Publishing, New Jersey,1990) 